\title{
Amylolytic glycoside hydrolases
}

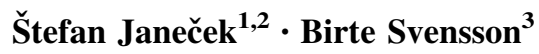

Received: 21 April 2016/Accepted: 22 April 2016/Published online: 29 April 2016

(C) Springer International Publishing 2016

Keywords Amylolytic enzymes - CAZy database . Starch and glycogen metabolism .

Structure and function of enzymes .

Protein design and evolution

When in 1991 Bernard Henrissat created the sequencebased classification of glycoside hydrolases (GH) [1], it was not easy to imagine at that time how huge scope and impact the subsequently established new database of Carbohydrate-Active enZymes, CAZy [2], would have. The $\alpha$ amylase family (family GH13) with 40 sequences bearing 10 different enzyme specificities was already from the beginning a bit exceptional compared to, e.g., the other amylolytic enzyme families of $\beta$-amylase and glucoamylase each encompassing just 5 sequences and no other specificities [1]. Currently, $\boldsymbol{\alpha}$-amylase, as a proper enzyme is present in the main $\alpha$-amylase family GH13 (forming with families GH70 and GH77 the clan GH-H), in families GH57 and GH119, and eventually in GH126 [3]. In addition to catalytic domains annotated to $\mathrm{GH}$ families, noncatalytic domains termed carbohydrate-binding modules

Štefan Janeček

Stefan.Janecek@savba.sk

1 Laboratory of Protein Evolution, Institute of Molecular Biology, Slovak Academy of Sciences, Dúbravská cesta 21, 84551 Bratislava, Slovakia

2 Department of Biology, Faculty of Natural Sciences, University of SS. Cyril and Methodius in Trnava, Nám. J. Herdu 2, 91701 Trnava, Slovakia

3 Enzyme and Protein Chemistry, Department of Systems Biology, Technical University of Denmark, Elektrovej Building 375, 2800 Kongens Lyngby, Denmark
(CBM) facilitating enzyme binding to different saccharides are also organised in sequence-based families as an integral part of the CAZy classification system [2].

The actual size of the main $\alpha$-amylase family GH13 with more than 31,500 members and 30 enzyme specificities is really impressive [2]. The overall concept of the $\alpha$-amylase family as it is known and accepted today was, however, based not only on GH classification, but resulted from contribution of an enormous amount of both experimental and theoretical studies. Among these pioneering prediction in silico analyses delivered by Birte Svensson and Ann MacGregor [4-9] have received special attention. In this regard, also the discovery of neopullulanase by Takashi Kuriki $[10,11]$, as a universal enzyme capable to catalyse all four main reactions characteristic for the family, i.e., hydrolysis and formation by transglycosylation of both $\alpha$-1,4- and $\alpha$-1,6-glycosidic linkages [12], deserves to be mentioned.

The present multi-author review issue was collected in an effort to illustrate and map the universe of amylolytic hydrolases and additional $\alpha$-glucan modifying enzymes with emphasis on retaining enzymes as well as other enzymes involved in starch biosynthesis, metabolism and oxidative degradation. The entire collection of eleven review articles starts with the story by the team led by Nicole Koropatkin on the Sus operon that constitutes the starch utilisation system in mammalian gut bacteria from the order Bacteroidetes and encodes eight unique proteins responsible for both binding and degradation of starch at the cell surface. The next two reviews written by the group of Birte Svensson and Eiji Suzuki and his co-author are devoted to structure/function and evolution of debranching and branching enzymes, respectively, from families GH13 and GH57 elucidating their nuances not only with regard to 
primary and tertiary structures but also within taxonomy. Two further reviews prepared by the groups of Magali Remaud-Simeon and Lubbert Dijkhuizen focus on the family GH70 glucansucrases presenting circularly permuted versions of the characteristic family GH13, the former article in addition pays attention to the biotechnologically important GH13 amylosucrase, while the latter highlights the very recent observation of non-permuted glucansucrases interestingly kept classified in family GH70. In the subsequent review, Stefan Janecek and his co-author offer details on three remarkable evolutionary stories concerning relatedness of plant and archaeal $\alpha$ amylases, animal proteins $\mathrm{rBAT}$ and $4 \mathrm{~F} 2$ and microbial $\alpha$ glucosidases-both from the family GH13 as well as the amylomaltases from borreliae of family GH77. The next article delivered by the team headed by Haruhide Mori and Atsuo Kimura illustrates diverse functions of $\alpha$-glucosidases from the main $\alpha$-amylase family GH13 compared to those from the related GH31 family that also includes $\alpha$ 1,4 -glucan lyases producing anhydrofructose as opposed to the $\alpha$-glucosidases which release $\alpha$-glucose.

The issue continues with the review written by the group of Joerg Fettke with focus on phosphorylation of glucosyl residues of starch during its metabolism mainly within green algae and land plants, which is mediated by two special enzymes called the glucan, water dikinase and the phosphoglucan, water dikinase containing also starchbinding domains of families CBM20 and CBM45. The following review prepared by the team of Matthew Gentry covers the glucan phosphatases SEX4 (and LSF2) and laforin responsible for dephosphorylation of starch in plants and glycogen in animals, respectively, consisting of a homologous dual-specificity phosphatase catalytic domain and, in the case of SEX4 and laforin, an additional domain of family CBM48 and CBM20, respectively. The following article by Samuel Zeeman and his co-author smoothly completes this topic and summarises current knowledge on the enzymatic machinery involved in insoluble starch granule biosynthesis in plant tissues with a special emphasis on specific functions these enzymes may play. The final review handled by Michael Marletta and his co-author brings details on starch-degrading lytic polysaccharide monooxygenases from auxiliary-activity family AA13 that degrade starch oxidatively via a copperdependent hydroxylation, boosting the activity of counterpart amylolytic enzymes of high interest in biotechnology.

\section{References}

1. Henrissat B (1991) A classification of glycosyl hydrolases based on amino acid sequence similarities. Biochem J 280:309-316

2. Cantarel BL, Coutinho PM, Rancurel C, Bernard T, Lombard V, Henrissat B (2009) The carbohydrate-active enzymes database (CAZy): an expert resource for glycogenomics. Nucleic Acids Res 37:D233-D238

3. Janecek S, Svensson B, MacGregor EA (2014) $\alpha$-Amylase-an enzyme specificity found in various families of glycoside hydrolases. Cell Mol Life Sci 71:1149-1170

4. MacGregor EA (1988) $\alpha$-Amylase structure and activity. J Protein Chem 7:399-415

5. Svensson B (1988) Regional distant sequence homology between amylases, $\alpha$-glucosidases and transglucanosylases. FEBS Lett 230:72-76

6. MacGregor EA, Svensson B (1989) A super-secondary structure predicted to be common to several $\alpha-1,4$-D-glucan-cleaving enzymes. Biochem J 259:145-152

7. Jespersen HM, MacGregor EA, Sierks MR, Svensson B (1991) Comparison of the domain-level organization of starch hydrolases and related enzymes. Biochem J 280:51-55

8. Jespersen HM, MacGregor EA, Henrissat B, Sierks MR, Svensson B (1993) Starch- and glycogen-debranching and branching enzymes:prediction of structural features of the catalytic $(\beta / \alpha)_{8^{-}}$ barrel domain and evolutionary relationship to other amylolytic enzymes. J Protein Chem 12:791-805

9. MacGregor EA, Jespersen HM, Svensson B (1996) A circularly permuted $\alpha$-amylase-type $\alpha / \beta$-barrel structure in glucan-synthesizing glucosyltransferases. FEBS Lett 378:263-266

10. Kuriki T, Imanaka T (1989) Nucleotide sequence of the neopullulanase gene from Bacillus stearothermophilus. J Gen Microbiol 135:1521-1528

11. Kuriki T, Takata H, Okada S, Imanaka T (1991) Analysis of the active center of Bacillus stearothermophilus neopullulanase. J Bacteriol 173:6147-6152

12. Takata H, Kuriki T, Okada S, Takesada Y, lizuka M, Minamiura N, Imanaka T (1992) Action of neopullulanase. Neopullulanase catalyzes both hydrolysis and transglycosylation at $\alpha-(1,4)-$ and $\alpha-(1,6)$-glucosidic linkages. J Biol Chem 267:18447-18452 